\title{
What we know about kininogen structure - importance for function and perspectives from cryo-EM
}

\author{
Michał B. Ponczek ${ }^{1}$
}

1 University of Lodz, Faculty of Biology and Environmental Protection, Department of General Biochemistry, Pomorska 141/143, 90-136 Lodz, Poland; michal.ponczek@biol.uni.lodz.pl

* Correspondence: michal.ponczek@biol.uni.lodz.pl; Tel.: ++48426354483

\begin{abstract}
Kininogens are multidomain glycoproteins found in the blood of most vertebrates. They are important in the blood coagulation cascade pathways - in intrinsic pathway activation leading to thrombin generation partially independently from tissue factor dependent extrinsic pathway, connecting blood coagulation with the kallikrein-kinin system. Nothing is known about the shape on atomic level therefore the endeavor to obtain the good-quality spatial structure of kininogens is important for a better understanding of their role in disease and treatment. Application of cryo-EM is important for solving the spatial structure of kininogens, drawing new frontiers in understanding the function and opening new pathways for drug development.
\end{abstract}

Keywords: kininogen; structure, AlphaFold, cryo-EM, proteins, kinin, bradykinin

\section{Introduction}

Kininogens or Fitzgerald factors are multidomain glycoproteins found in the blood of most vertebrates [1-4]. The orthological proteins, synonymic name kininogen-1, UniProt ID: P01042 for human, produced by splicing of the KNG1 gene transcript, is one of many proteins in the blood coagulation cascade pathways, in intrinsic pathway activation leading to thrombin generation partially independently from tissue factor (TF) extrinsic pathway, connecting blood coagulation with the kallikrein-kinin system (Figure 1). The proteins have been known and studied for decades, nonetheless, the spatial structure is still unknown. Three dimensional shape has so far not been solved, even fragmentarily, using crystallographic techniques or NMR for human ortholog nor for any other species, and there are still no coordinates in Protein Data Bank - PDB (Table 1).

Table 1. Blastp results of human HK amino acid sequence against Protein Data Bank proteins

\begin{tabular}{|c|c|c|}
\hline Description & Percent identity $^{1}$ & PDB Accession and chain 2 \\
\hline $\begin{array}{l}\text { Cleaved human fetuin-b in } \\
\text { complex with crayfish asta- } \\
\text { cin [Homo sapiens] }\end{array}$ & 26.20 & 6SAZ B \\
\hline $\begin{array}{c}\text { Crystal structure of L68V } \\
\text { mutant of human cystatin C } \\
{[\text { Homo sapiens }]}\end{array}$ & 29.73 & 3PS8 A \\
\hline $\begin{array}{c}\text { Human Cystatin C; Dimeric } \\
\text { Form With 3d Domain Swap- } \\
\text { ping [Homo sapiens] }\end{array}$ & 30.63 & $4 \mathrm{~N} 6 \mathrm{M} \mathrm{A}$ \\
\hline $\begin{array}{l}\text { Crystal structure of human } \\
\text { cystatin E/M [Homo sapiens] }\end{array}$ & 29.73 & $1 \mathrm{G} 96 \mathrm{~A}$ \\
\hline
\end{tabular}


Crystal structure of mono-

4N6L A meric human cystatin C stabilized against aggregation [Homo sapiens]

N-Truncated Human Cysta3GAX A tin C; Dimeric Form With 3D

Domain Swapping [Homo sapiens]

Crystal structure of V57G

1R4C A mutant of human cystatin $\mathrm{C}$ [Homo sapiens]

Crystal structure of V57P mutant of human cystatin $\mathrm{C}$ [Homo sapiens]

Hinge-loop mutation can be used to control 3D domain swapping and amyloidogenesis of human cystatin C [Homo sapiens]

${ }^{1}$ Percent identity is less than $30 \%$. Cystatin domain homology is detected. None is HK fragment (Date of search October 29, 2021)

${ }^{2}$ PDB according to https://www.rcsb.org/, https://www.ebi.ac.uk/pdbe/ and https://pdbj.org/.

Protein Basic Local Alignment Search Tool (BlastP) applied with human kininogen amino acid sequence against PDB returns only proteins containing homologous cystatin domains with identity about 30\%. Moreover, entering the keyword "kininogen" in the database search engine, in turn, returns only structures containing polypeptide fragments referred to as " inserts of kininogen peptides", "peptides derived from kininogen" or bradykinin (BK) derived peptides (Table 2).

Table 2. The best ten of 39 hits for searching "kininogen" keyword against RCSB PDB: https://www.rcsb.org/ (Date of search October 29, 2021)

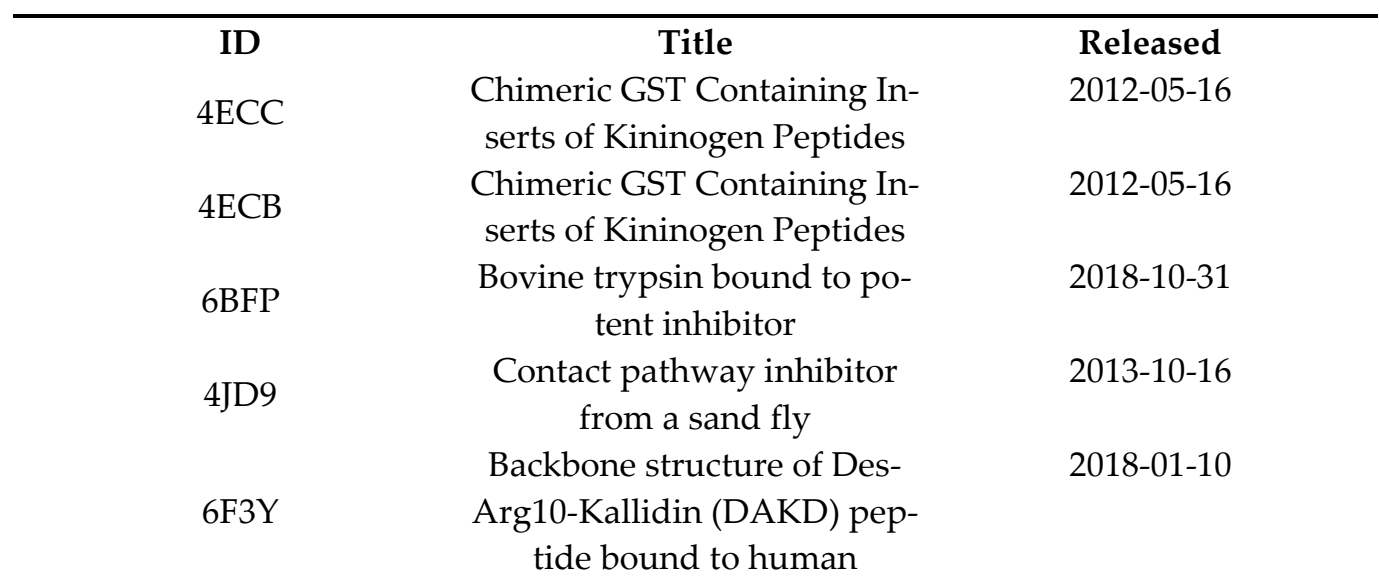


6 F3X

6F3W

6O1S

$6 \mathrm{O} 1 \mathrm{G}$

$6 \mathrm{~F} 27$
Bradykinin 1 Receptor (B1R) determined by DNP-enhanced MAS SSNMR

Backbone structure of DesArg10-Kallidin (DAKD) peptide in frozen DDM/CHS detergent micelle solution determined by DNP-enhanced MAS SSNMR

Backbone structure of free bradykinin (BK) in

DDM/CHS detergent micelle determined by MAS SSNMR

Structure of human plasma

kallikrein protease domain with inhibitor

Full length human plasma

kallikrein with inhibitor

NMR solution structure of

2018-01-10 non-bound [des-Arg10]kallidin (DAKD)
2018-01-10

2019-03-06

2019-03-06

2018-01-10

Therefore, the purpose of this review article is to elucidate the importance and prospects of the good-quality kininogens' spatial structure for a better understanding of their role in disease and treatment as we actually still know nothing about its shape on atomic level.

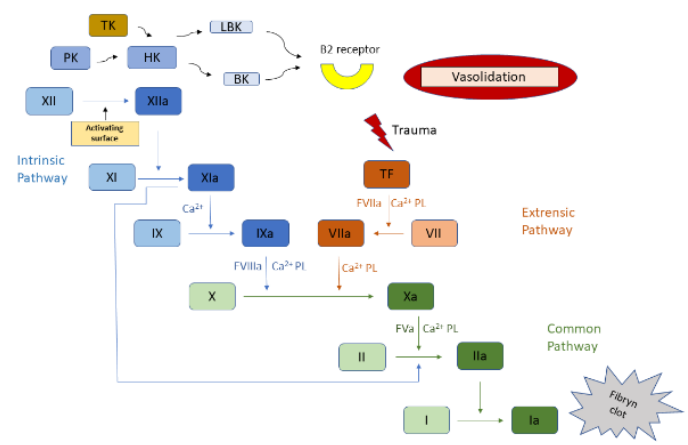

Figure 1. Blood coagulation with the kallikrein-kinin system. Roman numerals denote individual coagulation factors, the letter "a" stands for the active form, other abbreviations are as in the text.

\section{Kininogen biochemical and evolutionary characterization}

The human High Molecular Weight Kininogen $(\mathrm{HK})$ is a six domain glycoprotein with molecular weight of 108-120 kDa occurring in the blood plasma at a concentration approximately $70 \mu \mathrm{g} / \mathrm{mL}$, secreted into the blood by the liver, where the protein is mostly expressed in hepatocytes. Human HK theoretical isoelectric point based on amino acid sequence, according to Compute $\mathrm{pI} / \mathrm{Mw}$ tool (https://web.expasy.org/compute pi/) is 6.23 , the protein devoid of signal peptide counts 626 amino acid residues and the molecular mass is only $70 \mathrm{kDa}$, but because it undergoes numerous post-translational modifications, the real mass is much higher [1-4].

Kininogens, mostly $\mathrm{HK}$, have many different biological activities, including: the role in blood coagulation by helping to locate suitably plasma prekallikrein (PK) and factor XI 
(FXI) next to factor XII (FXII), the inhibition of blood platelets aggregation induced by thrombin and plasmin and generation of the active peptide BK released from HK. BK shows different important physiological effects: smooth muscle contraction, induction of hypotension, natriuresis and diuresis, and even decrease in blood glucose level. It is also a mediator of inflammation and causes and increase in vascular permeability, release of mediators of inflammation like prostaglandins, stimulation of nociceptors and has a direct (BK action) and indirect cardioprotective effect (by endothelium-derived relaxing factor action). Therefore, on the one hand the kininogens are important cofactors of blood coagulation, where autoactivation of FXII on a negatively charged surface leads to triggering the cascade through FXI, leading to contact (intrinsic) activation (Figure 1), on the other hand it is a source of BK - an extremely important vasodilation factor.

Kininogen is known to have a complex domain arrangement. Distinct domains of mature HK can be listed as follows with the amino acid positions numbering: Domain 1: 1-113, Domain 2: 114-234, Domain 3: 235-357, Domain 4: 358-383, Domain 5: 384-502, Domain 6: 503-626. The domains 1, 2, 3, 5 and 6 are roughly 120 amino acids in length and Domain 4 is only 26 amino acids long. Domains 1 and 6 are connected by a single disulfide bridge (Cys 10 to Cys 596) [4] (Figure 2).

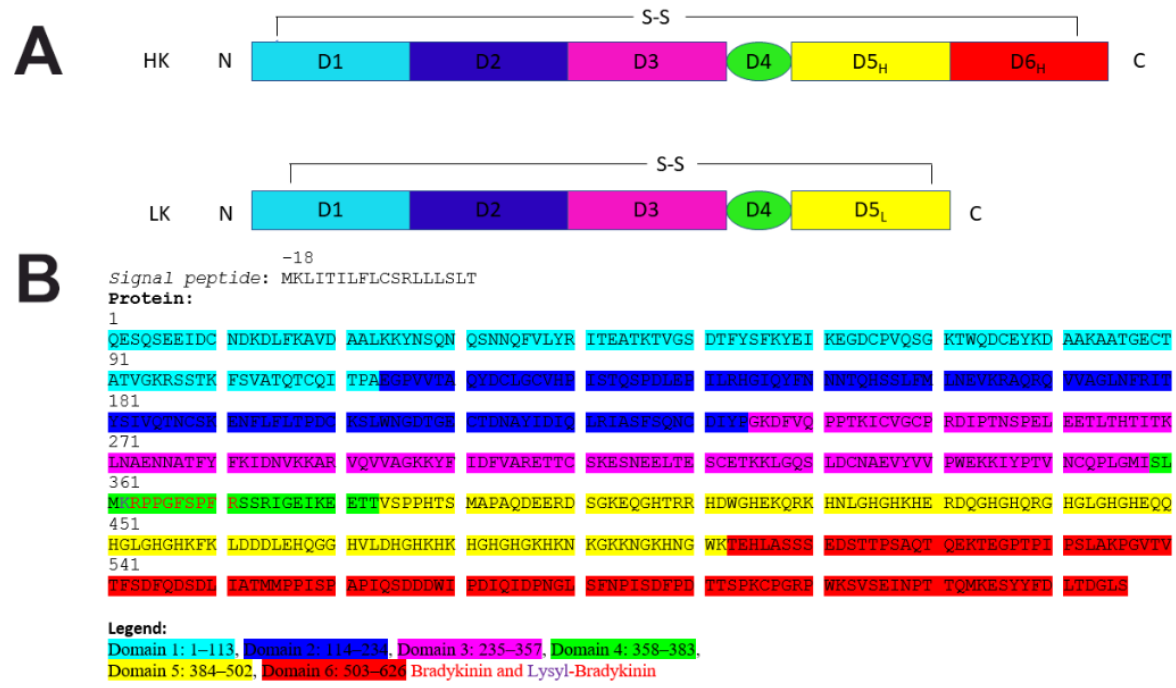

Figure 2. A. Human plasma kininogen alternative splicing products of messenger RNAs from the Kng1 gene - HK and LK. Both forms of protein have similar D1, D2, D3, and D4 domains, but different D5 domains, no Domain 6 is present in LK and ultimately the molecular weight of this form is lower than that of HK. B. Human HK amino acid sequence according to UniProt P01042 with the indication of domains. N-terminus and C-terminus marked by corresponding letters. Disulfide (SS) bonds between the respective domains are marked.

Some features and functions are recognized for these domains. Domains 1-3 belong to family of Cystatins (cysteine protease inhibitors, MEROPS inhibitor family I25, clan IH) [5-9] and can be simply detected based on human HK sequence using online databases of protein domains like PFAM (http://pfam.xfam.org/), PROSITE (https://prosite.expasy.org/) or SMART (http://smart.embl-heidelberg.de/). As cystatins-like domains their inhibitory action was demonstrated as follows: inhibition of atrial natriuretic factor (ANF) has been established in domain 1 and inhibition of calpain and papain (both cysteine or thiol proteases) has been combined with domains 2 and 3, respectively. The cleavage of HK in the smallest Domain 4 by activated plasma kallikrein (PK) releases nonapeptide bradykinin (RPPGFSPFR). BK boosts vasodilation and vascular permeability and can develop soft tissue swelling (angioedema) [4].

Domains 5 and 6 are vital for the contact pathway of blood coagulation. Domain 5 is rich in histidine motifs and binds to the negatively charged surfaces triggering the contact pathway together with coagulation factor XII (FXII). Domain 6 is responsible for binding 
of two related contact proteases - coagulation factor XI (FXI) and PK. Human HK can be divided into a heavy and a light chain after proteolytic cleavage in Domain 4 and reduction of disulfide bridge. The heavy chain spreads between amino acid residues 1-362 and contains domains 1-3 and the first six amino acids of the short Domain 4. The light chain is the rest of the molecule ( 255 acids) and consists of domains 5 and 6 . Both chains are glycosylated. There are known three $\mathrm{N}$-linked carbohydrates on the heavy chain and nine O-linked carbohydrates in the light chain. Messenger RNA alternative splicing transcript of the KNG1 gene is translated into a smaller protein variant $(70 \mathrm{kDa})$ - low molecular weight kininogen (LK). LK has identical domains 1-4 as HK albeit Domain 5 is shortened by 38 amino acids in length (Domain 5L) and Domain 6 is lacking. Digestion of LK by tissue kallikrein (TK) liberates the 10 amino acid peptide KRPPGFSPFR - Lysyl-Bradykinin (LBK) which is subsequently split to BK by the enzyme arginine aminopeptidase. LK is not involved in blood clotting, but inhibits the aggregation of blood platelets [4].

Thanks to genome and transcriptome sequencing it is nowadays known that a similar situation with KNG1 gene encoding alternatively spliced messenger RNAs for HK and LK occurs not only in human and other mammals, but also in reptiles, birds and amphibians, where HK and LK share D1 through D4 domains, but have varied D5 domains. D6 was found in HK from all tetrapods, suggesting HK combines PK in most terrestrial vertebrates. HK D5 is more mutable and in amphibians, crocodilians, birds and turtles it is shorter than in mammals and is absent in lizards and snakes. Cartilaginous or ray-finned fish kininogens are more similar to mammalian LK than to HK as D5 or fully formed D6 domains are nonexistent, although kininogens found in lobe-finned fishes (the coelacanth and the lungfish) have a D6 domain and short histidine-rich motifs corresponding to D5 domain. The appearance of HK in the lobe-finned fishes indicates an adaptation to blood clotting activation upon contact with silica-rich soil or mud impurities, the more so as FXII appears for the first time in the lungfishes, but disappears in the birds and the cetaceans where contact with the soil is much less frequent [10]. Evolutionary and functional links between orthological HK sequences can be enriched after solving the spatial structure for the human protein when used then as an adequate template for homology modelling.

\section{The importance of kininogen in civilization and infectious diseases}

HK significance, beyond physiological blood clotting, is participation in thrombotic disorders as it participates as cofactor of plasma coagulation factor XII (FXII) activation in contact blood clotting on many negatively charged surfaces (e.g. silica, nucleic acids, misfolded proteins, collagen, bacteria, or viruses). Thrombosis and embolism are still a significant problem connected to civilization diseases related to the circulatory system where the role of HK should not be neglected. Similarly, thrombotic complications and coagulation disorders appear frequently in infections with various pathogens where contact activation starts on pathological surfaces. The other side of the coin is BK released from HK. Coronavirus SARS-CoV-2 that spread causing the pandemic COVID-19 is known to bind Angiotensin-Converting Enzyme 2 (ACE2) and such binding increases BK release which promotes inflammation in lungs, causing cough and fever, as well as further activation of coagulation and the complement system. These systems are associated with angioedema, cardiovascular dysfunction, thrombosis, sepsis and disseminated intravascular coagulation (DIC). Emerging thrombotic complications in COVID-19 and reports of post-vaccination reactions indicate the need to investigate the relationship within the hemostatic system, including contact factors [11] within the interplay between renin-angiotensin, kallikrein-kinin and coagulation system activation during COVID infection [12]. The role of HK should not be overlooked here as a blood coagulation cofactor and a source of BK. Understanding the structure of $\mathrm{HK}$ is important for a better understanding of the changes and processes it undergoes not only in physiological state, but especially pathological situations like thrombotic disorders accompanying various civilization and infectious diseases, with the activation of the immune system, inflammation, and hemostasis. 


\section{Prospects for solving the spatial structure of kininogen}

Although images of HK were made many years ago by transmission electron microscopy (TEM) using various negative staining techniques, they do not shed much light on the actual shape of the kininogen [3,13,14]. In the oldest work from 1994 [3], where TEM images of human kininogen were taken, the samples were applied to a substrate from mica with tungsten shading and an $80 \mathrm{kV}$ Philips 400 microscope, magnification 60,000 was used. The protein preparation had a concentration of $2 \mathrm{mg}$ per $\mathrm{mL}, 0.15 \mathrm{M} \mathrm{NaCl}, \mathrm{pH}$ $5.30 .02 \mathrm{M}$ acetate. Using the scale provided by the authors, 3 domains of the kininogen seem to have 130-180 , and one domain is 50-90 $\AA$. In this work, together with HK, IgG antibodies (usually mass $150 \mathrm{kDa}$, length 100-150 $\mathrm{A}$ ) were visualized in some figures. Based on Figure $3 \mathrm{~b}$ from the cited paper, it can be calculated that the length of the antibody is about $150 \AA$, and the kininogen associated with this antibody is even $180 \AA$ long. Perhaps what the authors of this study showed are not individual kininogen molecules, but their aggregates, where a single domain is actually one kininogen molecule, the more as the antibody is clearly smaller at the picture with a higher molecular weight.

In a 2001 paper [13], the authors used $50 \mathrm{mM}$ Tris / HCl, pH 7.4 buffer in $150 \mathrm{mM}$ $\mathrm{NaCl}$, and used $0.75 \%$ uranyl formate for staining - two drops for $5 \mu \mathrm{L}$ of sample with a concentration of only 5-19 $\mu$ g per mL on glow discharged carbon-coated copper grids and used JEOL 1200 EX $60 \mathrm{kV}$ transmission electron microscope. In this work, according to the scale given in some figures, the protein ion-free molecule (EDTA) is 130-150 $\AA$, but one domain is $30-50 \AA$, in these individual domains, the substructure (as if subdomain) are visible. According to the authors, the molecule in the presence of $\mathrm{Zn}^{2+}$ is more compact and, according to the measurements corresponding to their scale, is about $100 \AA$, and the domains are 30-50 $\AA$. The authors themselves provide dimensions in the paper, estimating them at: $95 \pm 30 \AA$ (width) and $150 \pm 30 \AA$ (length) (paper Fig. 9B, C) - two larger domains $(55 \pm 20 \AA$ ) and three smaller ( $35 \pm 20 \AA$ ), and in the presence of zinc, according to them, the protein should be more spherical and compact, with dimensions reduced to $90 \pm 30 \AA$ (width) and $110 \pm 30 \AA$ (length) (cited paper Fig. 9D).

In the latest publication with TEM images of the kininogen from 2009 [14] according to the scale next to the TEM image (paper Fig. 1), the molecule corresponding to calculations based on the provided illustration should have only about $45 \AA$ diameter, and one can see the internal structures in the form of about 5-6 domains, where the given domain is only 15-20 $\AA$. The authors do not describe the TEM methodology in the paper, but only refer to an earlier work - the one from 2001 [13], and they do not provide numerical dimensions. In this study, the scale appears to underestimate the results.

In conclusion, based on the available cited above literature with published TEM results of kininogen, there is no clarity and consistency as to the size of this molecule and its domains. This additionally indicates the need for microscopic examination with newer techniques than those from more than decades ago.

Even though the primary sequence and the order of domains are well known, and some TEM images were taken many years ago, the spatial structure of this protein remains a mystery. The only results present currently in the PDB databases are polypeptide fragments derived from kininogen or BK bound to other proteins. The failure to publish the structure of HK may be due to the difficulty of its crystallization even in parts and inability to use NMR technique as the protein is too large. A certain light in the tunnel for 3D structure solution is AlphaFold Protein Structure Database, where draft HK structure was predicted by DeepMind calculations de novo (https://www.alphafold.ebi.ac.uk/entry/P01042) [15-18]. Unfortunately, despite the solution of cystatin domains (D1-D3) with good model confidence, the other domains of the protein are very low as almost unfolded floating chains and no glycans are present (Figure 3). 


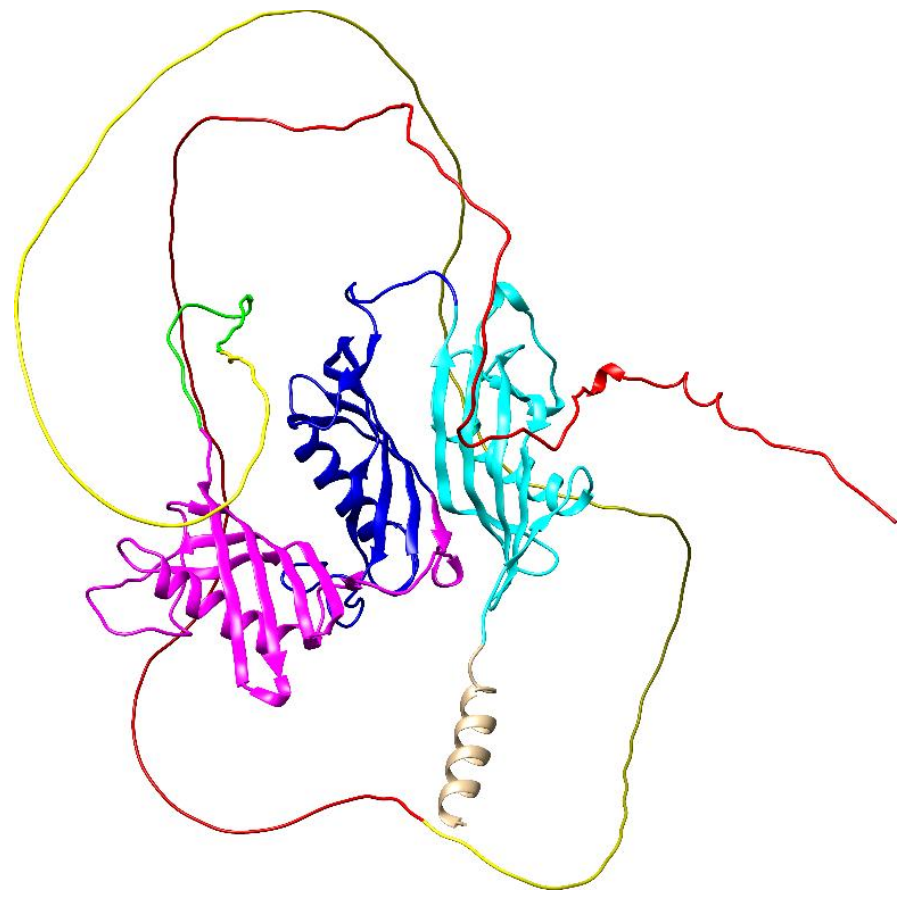

Figure 3. AlphaFold prediction of human HK. Colors of domains according to figure 2. The structure contains signal peptide colored brown.

A potential solution for this large $120 \mathrm{kDa}$ protein containing many sugar residues (theoretical molecular weight based only on the amino acid sequence is just $69896.70 \mathrm{Da}$ - own calculations based on the amino acid sequence on Compute $\mathrm{pI} / \mathrm{Mw}$ tool) is the use of cryogenic electron microscopy (cryo-EM). Protein crystallization is not necessary here and samples of protein solution are frozen to cryogenic temperatures by liquid ethane and fixed in an environment of thin film of vitreous ice to take electron microscope pictures of plenty protein molecules in different random orientations. Such flat two dimensional images are classified and processed by computer to generate three dimensional structure [19-21]. Nowadays available cryogenic microscopes with sensitive detectors and appropriate software enable to obtain a resolution for some proteins even $1.22 \AA$ using $300 \mathrm{kV}$ beam of electrons, although, unfortunately, the costs related to the equipment are still high [22-24]. More common and accessible dissemination of this technique should allow the solution of good-quality kininogen structures.

\section{Conclusions}

Kininogens are important proteins in blood circulation through the hemostatic system and the kallikrein-kinin system by the role in blood coagulation, helping to locate plasma PK and FXI next to FXII, by the inhibition of blood platelets aggregation induced by thrombin and plasmin and by generation of the active peptide BK released from HK. Different important physiological effects of BK can be listed: smooth muscle contraction, induction of hypotension, natriuresis and diuresis, decrease in blood glucose level, mediation of inflammation by release of prostaglandins, increase in vascular permeability, stimulation of nociceptors and cardioprotective effects. Despite the knowledge of the primary structure and the domain organization, still there are no known spatial structures of the whole proteins. Kininogens, playing a crucial role in many physiological and pathological processes related not only to blood clotting, but also to the regulation of the circulatory system due to vasodilation and the relationship with pressure regulation, through the interplay with renin-angiotensin systems, should not be neglected as far as the knowledge of the three-dimensional shape is concerned. Developed and improved visualization techniques with resolution at the atomic level should make this endeavor 
possible. Application of cryo-EM to determine the spatial structure of kininogens will draw new frontiers in understanding the function of these proteins and open new pathways for drug development.

Funding: This research received no external funding.

Institutional Review Board Statement: Not applicable

Informed Consent Statement: Not applicable

Data Availability Statement: Not applicable

\section{References}

1. Thompson, R.E.; Mandle, R. Jr; Kaplan, A.P. Characterization of human high molecular weight kininogen. Procoagulant activity associated with the light chain of kinin-free high molecular weight kininogen. J. Exp. Med. 1978, 147, 488-499.

2. Kerbiriou, D.M.; Griffin, J.H. Human high molecular weight kininogen. Studies of structure-function relationships and of proteolysis of the molecule occurring during contact activation of plasma. J. Biol. Chem. 1979, 254, 12020-12027.

3. Weisel, J.W.; Nagaswami, C.; Woodhead J.L.; DeLa Cadena, R.A.; Page J.D.; Colman, R.W. The shape of high molecular weight kininogen. Organization into structural domains, changes with activation, and interactions with prekallikrein, as determined by electron microscopy. J. Biol. Chem. 1994, 269, 10100-10106.

4. Winter, W.E.; Greene, D.N.; Beal, S.G.; Isom, J.A.; Manning, H.; Wilkerson G.; Harris N. Clotting factors: Clinical biochemistry and their roles as plasma enzymes. Adv. Clin. Chem. 2020, 94, 31-84.

5. Rawlings, N.D.; Barrett, A.J. Evolution of proteins of the cystatin superfamily. J. Mol. Evol. 1990, 30, 60-71.

6. Zhou, L.; Li-Ling, J.; Huang, H.; Ma, F.; Li, Q. Phylogenetic analysis of vertebrate kininogen genes. Genomics 2008, 91, 129-141.

7. Kordis, D.; Turk, V. Phylogenomic analysis of the cystatin superfamily in eukaryotes and prokaryotes. BMC Evol. Biol. 2009, 9, 266-266.

8. Zhou L.; Liu, X.; Jin, P.; Li, Q. Cloning of the kininogen gene from Lampetra japonica provides insights into its phylogeny in vertebrates. J. Genet. Genomics 2009, 36, 109-115.

9. Lalmanach, G.; Naudin, C.; Lecaille, F.; Fritz, H. Kininogens: More than cysteine protease inhibitors and kinin precursors. Biochimie 2010, 92, 1568-1579.

10. Ponczek, M.B.; Shamanaev, A.; LaPlace, A.; Dickeson, S.K.; Srivastava, P..; Sun, M.F.; Gruber, A., Kastrup, C.; Emsley, J.; Gailani D. The evolution of factor XI and the kallikrein-kinin system. Blood Adv. 2020, 4, 6135-6147.

11. Colarusso, C; Terlizzi, M.; Pinto, A.; Sorrentino, R. A lesson from a saboteur: High-MW kininogen impact in coronavirus-induced disease 2019, Br. J. Pharmacol. 2020, 177, 4866-4872.

12. Carvalho, P.R.; Sirois, P.; Fernandes; P.D. The role of kallikrein-kinin and renin-angiotensin systems in COVID-19 infection. Peptides 2021, 135, 170428.

13. Herwald, H.; Mörgelin, M.; Svensson, H.G.; Sjöbring, U. Zinc-dependent conformational changes in domain D5 of high molecular mass kininogen modulate contact activation. Eur. J. Biochem. 2001, 268, 396-404.

14. Oehmcke, S.; Mörgelin, M.; Herwald, H. Activation of the human contact system on neutrophil extracellular traps. J. Innate Immun. 2009, 1, 225-230.

15. AlQuraishi, M. AlphaFold at CASP13. Bioinformatics 2019, 35, 4862-4865.

16. Jumper J.; Evans R.; Pritzel A.; Green T.; Figurnov M.; Ronneberger O.; Tunyasuvunakool K.; Bates R.; Žídek A.; Potapenko A.; Bridgland, A.; Meyer, C.; Kohl, S.A.A.; Ballard, AJ.; Cowie, A.; Romera-Paredes, B.; Nikolov, S.; Jain, R.; Adler, J.; Back, T.; Petersen, S.; Reiman, D.; Clancy, E.; Zielinski, M.; Steinegger, M.; Pacholska, M.; Berghammer, T.; Bodenstein, S.; Silver, D.; Vinyals, O.; Senior, A.W.; Kavukcuoglu, K.; Kohli, P.; Hassabis, D. Highly accurate protein structure prediction with AlphaFold. Nature 2021, 596, 583-589.

17. Mullard A. What does AlphaFold mean for drug discovery? Nat. Rev. Drug. Discov. 2021, 20, 725-727.

18. Tong, A.B; Burch, J.D; McKay, D.; Bustamante. C.; Crackower, M.A.; Wu, H. Could AlphaFold revolutionize chemical therapeutics? Nat. Struct. Mol. Biol. 2021, 28, 771-772.

19. Burrows, N.D.; Penn, R.L. Cryogenic transmission electron microscopy: aqueous suspensions of nanoscale objects. Microsc. Microanal. 2013, 19, 1542-1553.

20. Earl, L.A.; Falconieri V.; Milne, J.L.; Subramaniam, S. Cryo-EM: beyond the microscope. Curr. Opin. Struct. Biol. $2017,46,71-78$.

21. Cheng, Y. Single-particle cryo-EM-How did it get here and where will it go. Science 2018, 361, 876-880.

22. Nakane, T.; Kotecha A.; Sente A.; McMullan G.; Masiulis S.; Brown P.M.G.E.; Grigoras I.T.; Malinauskaite L.; Malinauskas T.; Miehling J.; Uchański T.; Yu L.; Karia D.; Pechnikova E.V.; de Jong E.; Keizer J.; Bischoff M.; McCormack J.; Tiemeijer P.; 
Hardwick S.W.; Chirgadze D.Y.; Murshudov G.; Aricescu A.R.; Scheres S.H.W. Single-particle cryo-EM at atomic resolution. Nature 2020, 587, 152-156.

23. Yip K.M.; Fischer N.; Paknia E.; Chari A.; Stark H. Atomic-resolution protein structure determination by cryo-EM. Nature 2020. $587,157-161$.

24. Hand, E. Cryo-EM reveals exquisite molecular structures - at high cost. A cheaper microscope could bring the resolution revolution to the masses. Science 2020, 367, 354-358. 